\title{
Polarization distribution and degree of polarization for three-dimensional quantum light fields
}

\author{
Alfredo Luis* \\ Departamento de Óptica, Facultad de Ciencias Físicas, Universidad Complutense, 28040 Madrid, Spain
}

(Received 27 February 2005; published 22 June 2005)

\begin{abstract}
We introduce a probability distribution for polarization of three-dimensional quantum light fields as a marginal of the quadrature $Q$ function for a three-mode field by removing the variables irrelevant for polarization (total intensity and global phase). The probability distribution turns out to be determined by projection on $\mathrm{SU}(3)$ coherent states. We introduce a degree of polarization as the distance between the polarization distribution and the uniform distribution associated with completely unpolarized light. We study the relation between two- and three-dimensional polarization by considering field states with a component in the vacuum state. We apply this formalism to some relevant field states.
\end{abstract}

DOI: 10.1103/PhysRevA.71.063815

PACS number(s): 42.50.Dv, 03.65.Ca, 42.25.Ja

\section{INTRODUCTION}

Polarization is a fundamental ingredient of light, both in the quantum and in the classical domains. In the quantum regime this variable has been crucial for the experimental demonstration of fundamental issues such as entanglement, complementarity, quantum cryptography, teleportation, Bell inequalities, and precision measurements [1,2].

Although the electric field is by definition a threedimensional (3D) magnitude, most approaches to polarization assume two-dimensional (2D) transverse fields. This is because most problems in optics are addressed by considering plane-wave fields, where the longitudinal component vanishes. Moreover, polarization of harmonic waves is locally a two-dimensional phenomenon, since the electric field at each spatial point describes an ellipse contained in a plane.

Nevertheless, there are reasons justifying a threedimensional analysis of polarization. For example, for nonparaxial fields without a well-defined propagation direction the plane of the ellipse may vary from point to point. Also, plane-wave behavior and the vanishing of the longitudinal component are approximations [3]. Moreover, quantum fluctuations affect the three field components even when they are in the vacuum state, so that quantum polarization is perforce a $3 \mathrm{D}$ phenomenon $[4,5]$.

Up to now 3D polarization has been addressed by a generalization of the Stokes parameters and Stokes operators [5-13]. In this regard, some previous works have discussed the limitations of polarization formalisms based exclusively on the Stokes parameters [14]. These limitations arise because Stokes parameters are second-order field correlations, while higher-order correlations can be crucial, especially in quantum optics. For example, polarization squeezing is determined by the fluctuations of the Stokes operators rather than by their mean values $[2,15]$.

In this work we address a quantum description of 3D polarization beyond Stokes parameters by introducing a probability distribution for polarization as a $3 \mathrm{D}$ extension of

*Electronic address: alluis@ fis.ucm.es; URL: http://www.ucm.es/ info/gioq a previous 2D approach [14]. Quantum physics impels the statistical description of polarization from the very beginning. The complete specification of a definite polarization state would require precise values of the Stokes variables. In quantum optics they become noncommuting operators so that no state can have a definite value for all of them simultaneously and a probability distribution is required to describe every polarization state.

As a suitable probability distribution for polarization we propose the marginal of the quadrature $\mathcal{Q}$ function of a threemode field obtained after removing the variables irrelevant for the specification of the polarization state, i.e., the intensity and a global phase (Sec. II) [16]. We find that the polarization distribution for 3D fields is the projection of the state of the system on SU(3) coherent states [17] (Sec. II and Appendix B). Among other properties, the SU(3) coherent states are minimum uncertainty states for Stokes operators (Appendixes A and B) [5]. This supports their use as reference states for the definition of a polarization distribution.

Furthermore, we introduce a degree of polarization as the distance of the polarization distribution to the uniform distribution associated with completely unpolarized light (Sec. III). This approach goes beyond definitions based on the Stokes parameters since the distance between distributions involves field correlations of all orders. A similar approach has been adopted for the assessment of fringe visibility [18].

We examine also the relation between the $2 \mathrm{D}$ and $3 \mathrm{D}$ approaches by considering field states with a field component in vacuum (Sec. IV). Finally we consider some examples of relevant field states illustrating this formalism (Sec. V).

Finally, for the sake of completeness in Appendixes A and B we recall some previous results concerning generalized Stokes operators for three-dimensional fields and some properties of SU(3) coherent states.

\section{POLARIZATION DISTRIBUTION FOR THREE- DIMENSIONAL FIELDS}

In previous works dealing with quantum 2D polarization we have found it convenient to represent the statistical properties of field states by means of the quadrature $\mathcal{Q}$ function, 
defined by projection of the system state on quadrature coherent states [14]. This choice is motivated by the fact that, in sharp contrast to other quantum distributions known in physics, the quadrature $\mathcal{Q}$ function is always a true probability distribution and transforms properly under polarization changing transformations $[16,20]$. Moreover, the $\mathcal{Q}$ function can be determined in practice by simple experimental procedures whose principles of operation can be understood even within a purely classical framework [21]. To this end the schemes determining the Wigner function are also valid since $\mathcal{Q}$ is simply the convolution of the Wigner function with a Gaussian [16].

The 3D quadrature coherent states $|\boldsymbol{\alpha}\rangle$ with $a_{j}|\boldsymbol{\alpha}\rangle=\alpha_{j}|\boldsymbol{\alpha}\rangle$ are

$$
|\boldsymbol{\alpha}\rangle=e^{-\left(\left|\alpha_{1}\right|^{2}+\left|\alpha_{2}\right|^{2}+\left|\alpha_{3}\right|^{2}\right) / 2} \sum_{n_{1}, n_{2}, n_{3}=0}^{\infty} \frac{\alpha_{1}^{n_{1}} \alpha_{2}^{n_{2}} \alpha_{3}^{n_{3}}}{\sqrt{n_{1} ! n_{2} ! n_{3} !}}\left|n_{1}, n_{2}, n_{3}\right\rangle,
$$

with $\left|n_{1}, n_{2}, n_{3}\right\rangle$ being the number states and $a_{1}, a_{2}$, and $a_{3}$ the projection of the complex amplitude along Cartesian axes. The quadrature $\mathcal{Q}$ function is [16]

$$
\mathcal{Q}(\boldsymbol{\alpha})=\frac{1}{\pi^{3}} \operatorname{tr}(\rho|\boldsymbol{\alpha}\rangle\langle\boldsymbol{\alpha}|),
$$

where $\rho$ is the density matrix.

We can derive a probability distribution for polarization if we remove from $\mathcal{Q}$ the degrees of freedom irrelevant for the description of polarization. These are the total intensity $\left|\alpha_{1}\right|^{2}+\left|\alpha_{2}\right|^{2}+\left|\alpha_{3}\right|^{2}$ and a global phase, since only relative intensities and relative phases enter on the idea of polarization. After this removal we will get a distribution depending on four real variables. This is consistent with the fact that the specification of a classical polarization state in a threedimensional space requires four parameters: Two define the plane of the ellipse, and the other two fix the ellipse.

In order to remove the total intensity and the global phase it is convenient to express $\boldsymbol{\alpha}$ in terms of phase-angular coordinates in the form

$$
\begin{gathered}
\alpha_{1}=r \sin \frac{\theta^{\prime}}{2} \sin \frac{\theta}{2} e^{i\left(\delta-\phi^{\prime} / 2-\phi / 2\right)}, \\
\alpha_{2}=r \sin \frac{\theta^{\prime}}{2} \cos \frac{\theta}{2} e^{i\left(\delta-\phi^{\prime} / 2+\phi / 2\right)} \\
\alpha_{3}=r \cos \frac{\theta^{\prime}}{2} e^{i\left(\delta+\phi^{\prime} / 2+\phi / 2\right)}
\end{gathered}
$$

where $\infty \geqslant r \geqslant 0, \pi \geqslant \theta, \theta^{\prime} \geqslant 0$, and $2 \pi \geqslant \delta, \phi, \phi^{\prime} \geqslant 0$.

The Jacobian of the transformation leads to

$$
d^{2} \alpha_{1} d^{2} \alpha_{2} d^{2} \alpha_{3}=\frac{1}{32} r^{5} d r d \delta d \Gamma,
$$

being

$$
d \Gamma=4 \sin \theta \cos \frac{\theta^{\prime}}{2} \sin ^{3} \frac{\theta^{\prime}}{2} d \theta d \theta^{\prime} d \phi d \phi^{\prime},
$$

with

$$
\int d \Gamma=(4 \pi)^{2},
$$

and $d^{2} \alpha=d \alpha_{r} d \alpha_{i}$, with $\alpha_{r}, \alpha_{i}$ being the real and imaginary parts of $\alpha$, respectively.

With this change of variables, the quadrature coherent states $|\boldsymbol{\alpha}\rangle$ can be expressed as

$$
|\boldsymbol{\alpha}\rangle=e^{-r^{2} / 2} \sum_{N=0}^{\infty} \frac{\left[r e^{i\left(\delta+\phi^{\prime} / 2+\phi / 2\right)}\right]^{N}}{\sqrt{N !}}|N, \Gamma\rangle,
$$

where $|N, \Gamma\rangle$ are $\mathrm{SU}(3)$ coherent states [17],

$$
\begin{aligned}
|N, \Gamma\rangle= & \sum_{n=0}^{N}\left(\begin{array}{l}
N \\
n
\end{array}\right)^{1 / 2}\left(\cos \frac{\theta^{\prime}}{2}\right)^{N-n}\left(\sin \frac{\theta^{\prime}}{2}\right)^{n} \\
& e^{-i n \phi^{\prime}}|n, \Omega\rangle_{1,2}|N-n\rangle_{3},
\end{aligned}
$$

$|n, \Omega\rangle_{1,2}$ are $\mathrm{SU}(2)$ coherent states in modes $a_{1}, a_{2}$ [22],

$$
|n, \Omega\rangle_{1,2}=\sum_{m=0}^{n}\left(\begin{array}{l}
n \\
m
\end{array}\right)^{1 / 2}\left(\cos \frac{\theta}{2}\right)^{n-m}\left(\sin \frac{\theta}{2}\right)^{m} e^{-i m \phi}|m\rangle_{1}|n-m\rangle_{2},
$$

and $|N-n\rangle_{3},|m\rangle_{1},|n-m\rangle_{2}$ are number states in the corresponding modes. In Appendix B we provide further details and properties of the states $|N, \Gamma\rangle$.

The removal of $r$ and $\delta$ in Eq. (2.2) leads to the $Q$ function for polarization variables,

$$
Q(\Gamma)=\frac{1}{32} \int_{0}^{\infty} d r \int_{0}^{2 \pi} d \delta r^{5} \mathcal{Q}(\boldsymbol{\alpha})=\operatorname{tr}[\rho \Delta(\Gamma)],
$$

where

$$
\begin{aligned}
\Delta(\Gamma) & =\frac{1}{32 \pi^{3}} \int_{0}^{\infty} d r \int_{0}^{2 \pi} d \delta r^{5}|\boldsymbol{\alpha}\rangle\langle\boldsymbol{\alpha}| \\
& =\sum_{N=0}^{\infty} \frac{(N+2)(N+1)}{2(4 \pi)^{2}}|N, \Gamma\rangle\langle N, \Gamma|
\end{aligned}
$$

satisfies the resolution of the identity

$$
\int d \Gamma \Delta(\Gamma)=I,
$$

where $I$ is the identity.

This procedure defines a legitimate probability distribution $Q(\Gamma)$ which is nonnegative $Q(\Gamma) \geqslant 0$ and normalized $\int d \Gamma Q(\Gamma)=1$. We can appreciate that $Q(\Gamma)$ is defined by projection of the field state on the SU(3) coherent states. There are reasons that make this fact attractive and consistent with previous results. In Ref. [5] it is shown that the $\mathrm{SU}(3)$ coherent states $|N, \Gamma\rangle$ are the minimum uncertainty states for the Stokes operators with fixed total number of photons. Moreover, they are also maximally polarized states when the de- 
gree of polarization is measured in terms of the Stokes parameters [5]. Therefore the SU(3) coherent states can be regarded as the quantum states closer to represent a definite three-dimensional polarization state with fixed intensity.

The SU(3) and SU(2) coherent states can also be related to an operational approach to quantum polarization. In practical terms, we may say that the output of a linear polarizer (all the photons in a single mode and the rest of modes in vacuum) is as much polarized as allowed by the quantum theory. Then we can construct any other quantum polarized state by applying rotations and phase plates (i.e., arbitrary energy-conserving linear transformations). This leads precisely to the $\mathrm{SU}(2)$ coherent states in a $2 \mathrm{D}$ approach and to the $\mathrm{SU}(3)$ coherent states in a 3D approach.

This operational perspective agrees with the scheme for a direct determination of the polarization distribution $Q(\Gamma)$ that can be derived from the approaches analyzed in Ref. [23] in the context of spin systems. The idea is to measure the probability that all the photons are in a single mode after a SU(2) or SU(3) transformation. Some other similar procedures based on the measurement of the mode intensities after $\mathrm{SU}(2)$ or SU(3) transformations can be found in Ref. [24]. All these approaches allow the determination of $Q(\Gamma)$ without determining first the quadrature distribution $\mathcal{Q}(\boldsymbol{\alpha})$, i.e., avoiding the complete reconstruction of the state of the system.

In the above analysis we have assumed the minimum number of modes (three) required to describe a threedimensional field. However, there would be situations where a larger number of modes can contribute to the field, for example when multiple beams cross the point of interest propagating in different directions. Next we show in brief that there is a generalization of the above approach to encompass such multimode situations. The starting point is provided by multimode quadrature coherent states $\left|\boldsymbol{\alpha}_{1}, \boldsymbol{\alpha}_{2}, \boldsymbol{\alpha}_{3}\right\rangle$ defining a quadrature distribution $\mathcal{Q}\left(\boldsymbol{\alpha}_{1}, \boldsymbol{\alpha}_{2}, \boldsymbol{\alpha}_{3}\right)$, where the three multi-dimensional complex vectors $\boldsymbol{\alpha}_{j}, j=1$, 2,3 , denote the complex amplitudes of the coherent states of the modes contributing to the component $j$ of the field. The relevant variables are the three components of the electric field along three Cartesian axes at the point of interest. These projections $\alpha_{j}$ are given by the sums $\alpha_{j}=\Sigma_{\ell} \alpha_{j, \ell}$ where $\alpha_{j, \ell}$ are the components of $\boldsymbol{\alpha}_{j}$. In a first stage we can carry out a first removal of variables irrelevant for the specification of polarization by averaging $\mathcal{Q}\left(\boldsymbol{\alpha}_{1}, \boldsymbol{\alpha}_{2}, \boldsymbol{\alpha}_{3}\right)$ over all the degrees of freedom different from $\boldsymbol{\alpha}=\left(\alpha_{1}, \alpha_{2}, \alpha_{3}\right)$. The details of the procedure will depend on the particular number of modes. The result is an effective three-mode quadrature distribution $\mathcal{Q}(\boldsymbol{\alpha})$. In a second stage we can perform the final removal from $\mathcal{Q}(\boldsymbol{\alpha})$ of the variables irrelevant for the specification of polarization as described in Eqs. (2.3) and (2.10).

\section{DEGREE OF POLARIZATION FOR THREE- DIMENSIONAL FIELDS}

The above polarization distribution provides a very natural measure $\mathcal{D}$ of the degree of polarization for 3D fields as the distance between $Q$ and the uniform distribution associated with fully unpolarized light $[14,25]$,

$$
Q_{\text {unpolarized }}(\Gamma)=\frac{1}{(4 \pi)^{2}},
$$

as

$$
\mathcal{D}=(4 \pi)^{2} \int d \Gamma\left[Q(\Gamma)-\frac{1}{(4 \pi)^{2}}\right]^{2}=(4 \pi)^{2} \int d \Gamma Q^{2}(\Gamma)-1 .
$$

It must be noticed that $\mathcal{D}$ is not bounded $\infty>\mathcal{D} \geqslant 0$. If a normalized degree would be required we might consider expressions such as $\mathcal{D} /(1+\mathcal{D})$, for example.

The inverse of $\int d \Gamma Q^{2}(\Gamma)$ is a measure of the volume of the polarization space occupied by the function $Q$, so that the smaller the volume over which $Q$ extends, the larger the degree of polarization. A similar strategy has been adopted in different areas $[18,26,27]$.

According to Ref. [27] the $\mathrm{SU}(3)$ coherent states $|N, \Gamma\rangle$ are the states with larger $\int d \Gamma Q^{2}(\Gamma)$ for fixed $N$. In our context, this implies that $|N, \Gamma\rangle$ are the states with the maximum degree of polarization $\mathcal{D}$ for fixed intensity. The explicit calculation of $\mathcal{D}$ for $|N, \Gamma\rangle$ is carried out in Sec. V.

Next we show that $\mathcal{D}$ is invariant under linear energyconserving transformation $U$ of the complex amplitudes

$$
U^{\dagger} a_{j} U=\sum_{k=1}^{3} \mathcal{U}_{j, k} a_{k},
$$

where $\mathcal{U}_{j, k}$ is a unitary matrix. This transformation maps coherent states into coherent states with different complex amplitudes $\widetilde{\boldsymbol{\alpha}}=\mathcal{U} \boldsymbol{\alpha}$ preserving the volume element

$$
d^{2} \widetilde{\alpha}_{1} d^{2} \widetilde{\alpha}_{2} d^{2} \widetilde{\alpha}_{3}=d^{2} \alpha_{1} d^{2} \alpha_{2} d^{2} \alpha_{3},
$$

since this is a linear canonical transformation. The idea is to use Eq. (2.4) to transform this into the same equality for the polarization volume element. To this end we note that $\tilde{r}=r$. Concerning the transformation of the $\delta$ variable, from Eq. (2.3) we get

$$
\widetilde{\delta}=\delta+f(\Gamma, \mathcal{U}),
$$

where $f$ is a function of the polarization variables and the transformation. This relation holds because $\widetilde{\boldsymbol{\alpha}}$ is just a function of $\boldsymbol{\alpha}$ but not of $\boldsymbol{\alpha}^{*}$. Since $\tilde{\delta}$ embraces $\delta$ completely, the other transformed polarization variables $\widetilde{\Gamma}=\tilde{\theta}, \widetilde{\theta^{\prime}}, \widetilde{\phi}, \widetilde{\phi}^{\prime}$ do no depend on $\delta$,

$$
\frac{\partial \widetilde{\Gamma}}{\partial \delta}=0
$$

These vanishing derivatives along with $\tilde{r}=r$ imply the following for the Jacobian of the transformation of the polarization variables

$$
\frac{\partial \widetilde{\boldsymbol{\alpha}}}{\partial \boldsymbol{\alpha}}=\frac{\partial(\widetilde{r}, \tilde{\delta}, \widetilde{\Gamma})}{\partial(r, \delta, \Gamma)}=\frac{\partial \widetilde{\Gamma}}{\partial \Gamma}=1,
$$

so that $d \Gamma=d \widetilde{\Gamma}$. This in turn implies 


$$
\int d \Gamma Q_{U \rho U^{\dagger}}^{2}(\Gamma)=\int d \Gamma Q_{\rho}^{2}(\widetilde{\Gamma})=\int d \Gamma Q_{\rho}^{2}(\Gamma),
$$

that demonstrates the above-mentioned invariance of the degree of polarization

$$
\mathcal{D}_{U \rho U^{\dagger}}=\mathcal{D}_{\rho},
$$

valid for linear energy-conserving transformations $U$.

Concerning the practical determination of the degree of polarization, up to now we have found no practical procedure for a direct measurement of $\mathcal{D}$. The only method currently available consists in the evaluation of the integration in Eq. (3.2) after the measurement of the polarization distribution $Q(\Gamma)$, for example as discussed in Sec. II.

\section{RELATION BETWEEN THE THREE-DIMENSIONAL AND TWO-DIMENSIONAL DEGREES OF POLARIZATION}

It is natural to ask whether there exists a definite relation between two- and three-dimensional approaches to quantum polarization. To this end we should consider field states as close as possible to have a vanishing component, say $a_{3}$, by assuming that the mode $a_{3}$ is in the vacuum state $|0\rangle_{3}$,

$$
\rho_{3 \mathrm{D}}=\rho_{2 \mathrm{D}} \otimes|0\rangle_{33}\langle 0| \text {. }
$$

We can update the $3 \mathrm{D}$ polarization distribution by projecting the positive operator measure (2.11) on $|0\rangle_{3}$,

$$
{ }_{3}\langle 0 \mid N, \Gamma\rangle\langle N, \Gamma \mid 0\rangle_{3}=\left(\sin \frac{\theta^{\prime}}{2}\right)^{2 N}|N, \Omega\rangle\langle N, \Omega|,
$$

where $|N, \Omega\rangle$ is a $\mathrm{SU}(2)$ coherent state (2.9), leading to

$$
Q_{3 \mathrm{D}}(\Gamma)=\sum_{N=0}^{\infty} \frac{(N+2)(N+1)}{2(4 \pi)^{2}}\left(\sin \frac{\theta^{\prime}}{2}\right)^{2 N}\left\langle N, \Omega\left|\rho_{2 \mathrm{D}}\right| N, \Omega\right\rangle .
$$

This shows that the 2D distribution can be readily obtained from the $3 \mathrm{D}$ counterpart as a marginal by removing the $\theta^{\prime}, \phi^{\prime}$ dependence

$$
\begin{aligned}
Q_{2 \mathrm{D}}(\Omega) & =\sum_{N=0}^{\infty} \frac{N+1}{4 \pi}\left\langle N, \Omega\left|\rho_{2 \mathrm{D}}\right| N, \Omega\right\rangle \\
& =4 \int d \theta^{\prime} d \phi^{\prime} \cos \frac{\theta^{\prime}}{2} \sin ^{3} \frac{\theta^{\prime}}{2} Q_{3 \mathrm{D}}(\Gamma),
\end{aligned}
$$

where the trigonometric functions come from the volume element (2.5).

A closer relationship between $Q_{3 \mathrm{D}}(\Gamma)$ and $Q_{2 \mathrm{D}}(\Omega)$ can only be obtained if we focus on field states with a definite number of photons in modes $a_{1}, a_{2}$. In such a case, for fixed $N$ we get

$$
Q_{3 \mathrm{D}}(\Gamma)=\frac{N+2}{8 \pi}\left(\sin \frac{\theta^{\prime}}{2}\right)^{2 N} Q_{2 \mathrm{D}}(\Omega) .
$$

Next we inquire about the relation between the degree of $3 \mathrm{D}$ polarization $\mathcal{D}_{3 \mathrm{D}}$ that naturally includes the fluctuations of the third dimension, and its $2 \mathrm{D}$ counterpart $\mathcal{D}_{2 \mathrm{D}}[14]$,

$$
\mathcal{D}_{2 \mathrm{D}}=4 \pi \int d \Omega Q_{2 \mathrm{D}}^{2}(\Omega)-1,
$$

that ignores the fluctuations of the third dimension from the very beginning.

Focusing again for simplicity in the case of fixed total photon number, we get from Eq. (4.5) that

$$
\int d \Gamma Q_{3 \mathrm{D}}^{2}(\Gamma)=\frac{1}{16 \pi} \frac{(N+2)^{2}}{(N+1)} \int d \Omega Q_{2 \mathrm{D}}^{2}(\Omega),
$$

and then

$$
\mathcal{D}_{3 \mathrm{D}}=\frac{(N+2)^{2}}{4(N+1)} \mathcal{D}_{2 \mathrm{D}}+\frac{N^{2}}{4(N+1)} .
$$

We obtain $\mathcal{D}_{3 \mathrm{D}}>\mathcal{D}_{2 \mathrm{D}}$ (leaving aside the case $N=0$ which corresponds to the vacuum state for which $\mathcal{D}_{3 \mathrm{D}}=\mathcal{D}_{2 \mathrm{D}}=0$ ). This result can be explained qualitatively as follows: When a fluctuating two-dimensional field is embedded in a larger dimension the relative amount of uncertainty must decrease since we know in advance that one of the field components vanishes $[5,10]$. For example, two random uncorrelated field components of the same intensity are fully unpolarized light in a two-dimensional space but this is certainly not completely unpolarized light in three dimensions, since this would require three random uncorrelated components of the same intensity. This agrees with a similar analysis in terms of Stokes parameters $[5,10]$.

\section{SOME EXAMPLES}

Next we apply the above definitions to some relevant field states.

\section{A. One-photon states}

The Hilbert space for one photon split between three modes is three-dimensional, being spanned by the photonnumber eigenstates $|1,0,0\rangle,|0,1,0\rangle$, and $|0,0,1\rangle$. Taking this as a basis, any density matrix can be expanded in terms of the Gell-Mann matrices (Appendix B) [6,8-11]

$$
\rho=\frac{1}{3}\left\langle S_{0}\right\rangle \lambda_{0}+\frac{1}{2} \sum_{j=1}^{8}\left\langle S_{j}\right\rangle \lambda_{j},
$$

where the coefficients of this expansion are the Stokes parameters. For $N=1$ the states $|N, \Gamma\rangle$ are

$$
\begin{aligned}
|1, \Gamma\rangle= & \cos \frac{\theta^{\prime}}{2}|0,0,1\rangle+\cos \frac{\theta}{2} \sin \frac{\theta^{\prime}}{2} e^{-i \phi^{\prime}}|0,1,0\rangle \\
& +\sin \frac{\theta}{2} \sin \frac{\theta^{\prime}}{2} e^{-i\left(\phi+\phi^{\prime}\right)}|1,0,0\rangle .
\end{aligned}
$$

The $Q$ function for the state $(5.1)$ is

$$
Q(\Gamma)=\frac{1}{(4 \pi)^{2}}\left(1+\frac{3}{2} \sum_{j=1}^{8}\left\langle S_{j}\right\rangle \mu_{j}(\Gamma)\right),
$$

where the $\mu_{j}(\Gamma)$ functions are defined in Eq. (B9). This leads to 


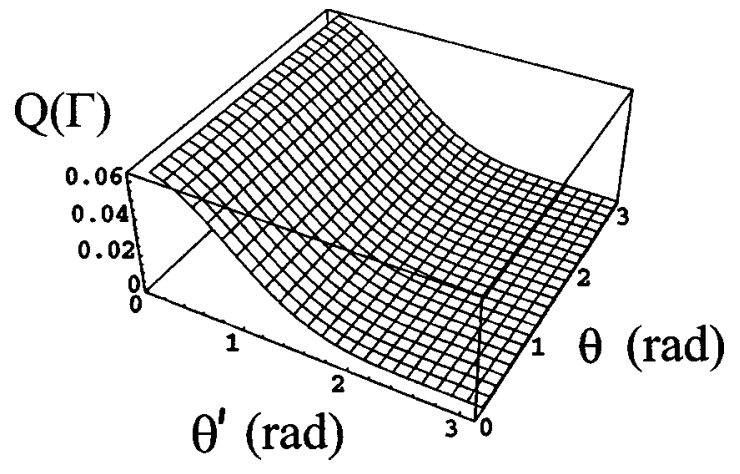

FIG. 1. $Q(\Gamma)$ as a function of $\theta$ and $\theta^{\prime}$ for a number state with $n_{1}=n_{2}=0, n_{3}=3$.

$$
\mathcal{D}=\frac{3}{8} \sum_{j=1}^{8}\left\langle S_{j}\right\rangle^{2}
$$

This suggests that there should be a direct relation between $\mathcal{D}$ and the degree of polarization $P$ defined in terms of the Stokes parameters and recalled in Eq. (A6) [5]. Using Eq. (A6) and taking into account that the state (5.1) is an eigenstate of the total photon number $S_{0}$, we get $\mathcal{D}=2 P^{2}$. This relation arises in this case because every one-photon state is fully specified by the Stokes parameters. Such a relationship no longer holds for larger photon numbers.

\section{B. Number states}

For the number states $\left|n_{1}, n_{2}, n_{3}\right\rangle$ we get

$$
\begin{aligned}
Q(\Gamma)= & \frac{1}{32 \pi^{2}} \frac{\left(n_{1}+n_{2}+n_{3}+2\right) !}{n_{1} ! n_{2} ! n_{3} !}\left(\sin \frac{\theta}{2} \sin \frac{\theta^{\prime}}{2}\right)^{2 n_{1}} \\
& \times\left(\cos \frac{\theta}{2} \sin \frac{\theta^{\prime}}{2}\right)^{2 n_{2}}\left(\cos \frac{\theta^{\prime}}{2}\right)^{2 n_{3}},
\end{aligned}
$$

leading to

$$
\mathcal{D}=\frac{1}{2}\left[\frac{\left(n_{1}+n_{2}+n_{3}+2\right) !}{n_{1} ! n_{2} ! n_{3} !}\right]^{2} \frac{\left(2 n_{1}\right) !\left(2 n_{2}\right) !\left(2 n_{3}\right) !}{\left(2 n_{1}+2 n_{2}+2 n_{3}+2\right) !}-1 .
$$

It can be seen that for fixed $N=n_{1}+n_{2}+n_{3}$ the maximum of $\mathcal{D}$ is obtained when all the photons are in the same mode, (i. e., $|0,0, N\rangle,|0, N, 0\rangle$, or $|N, 0,0\rangle$ ), with

$$
\mathcal{D}=\frac{N^{2}(N+5)}{4(2 N+1)}
$$

that for large $N$ scales as $\mathcal{D} \simeq N^{2} / 8$. Actually, the states $|0,0, N\rangle,|0, N, 0\rangle$, and $|N, 0,0\rangle$ are $\mathrm{SU}(3)$ coherent states with $\theta^{\prime}=0$ for the first one, $\theta^{\prime}=\pi, \theta=0$ for the second one, and $\theta^{\prime}=\pi, \theta=\pi$ for the last one.

In Figs. 1 and 2, we have represented $Q(\Gamma)$ for $n_{1}=n_{2}$ $=0, n_{3}=3$, and $n_{1}=3, n_{2}=n_{3}=0$, respectively.

On the other hand, the minimum $\mathcal{D}$ occurs for the equipartition state $|n, n, n\rangle$ with the same number of photons in all modes $n_{1}=n_{2}=n_{3}=n$. This example is interesting since all the Stokes parameters vanish $\left\langle S_{j}\right\rangle=0$ for $j=1, \ldots, 8$. However,

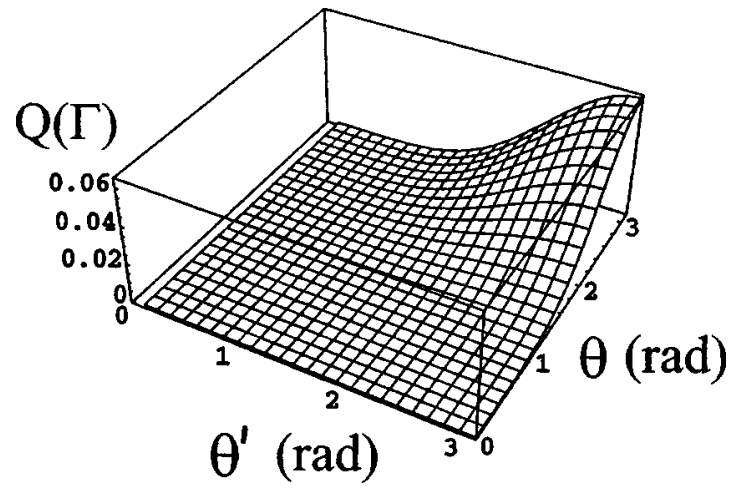

FIG. 2. $Q(\Gamma)$ as a function of $\theta$ and $\theta^{\prime}$ for a number state with $n_{1}=3, n_{2}=n_{3}=0$.

this is not an unpolarized state since the polarization distribution is far from being uniform as illustrated by Fig. 3. This is conveniently reflected by the degree of polarization (5.6), and for large $n$ we get

$$
\mathcal{D} \simeq \frac{3 \sqrt{3}}{8 \pi} N
$$

where $N=3 n$ is the total number of photons. The degree of polarization $\mathcal{D}$ never vanish, but increases with the number of photons. Nevertheless, this growing is lesser than for the states $|0,0, N\rangle,|0, N, 0\rangle$, and $|N, 0,0\rangle$.

\section{C. $\mathrm{SU}(3)$ coherent states}

The SU(3) coherent states are obtained from the photon number state $|0,0, N\rangle$ by a linear energy-conserving transformation as recalled in Appendix B. Therefore we can apply the invariance of $\mathcal{D}$ under linear energy-conserving transformations discussed in Sec. III to conclude that all the SU(3) coherent states with the same $N$ have the same degree of polarization in Eq. (5.7).

\section{Quadrature coherent states}

By using again the invariance of $\mathcal{D}$ under linear energyconserving transformations we can consider without loss of generality the quadrature coherent state $|0,0, \alpha\rangle$ which is a

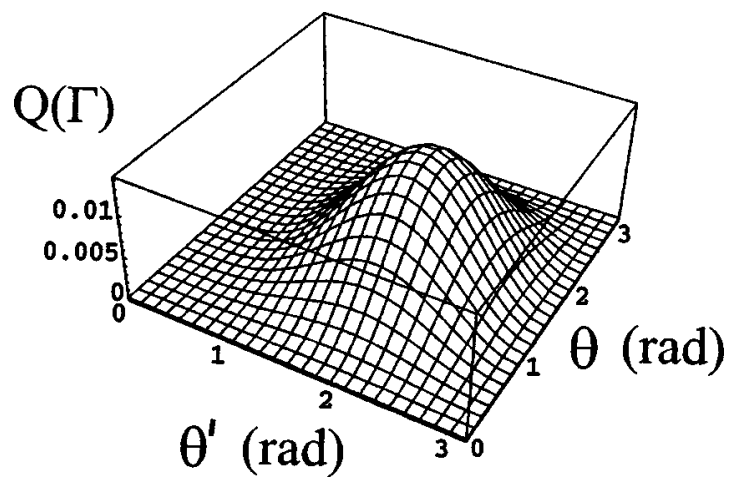

FIG. 3. $Q(\Gamma)$ as a function of $\theta$ and $\theta^{\prime}$ for a number state with $n_{1}=n_{2}=n_{3}=1$. 
Poissonian superposition of the number states $|0,0, N\rangle$,

$$
Q(\Gamma)=\frac{1}{32 \pi^{2}} \sum_{N=0}^{\infty}(N+2)(N+1) \frac{\bar{n}^{N}}{N !} e^{-\bar{n}} \cos ^{2 N} \frac{\theta^{\prime}}{2},
$$

where $\bar{n}=|\alpha|^{2}$ is the mean number of photons. The sum can be performed leading to

$$
Q(\Gamma)=\frac{1}{32 \pi^{2}}\left[\bar{n}^{2} \cos ^{4} \frac{\theta^{\prime}}{2}+4 \bar{n} \cos ^{2} \frac{\theta^{\prime}}{2}+2\right] e^{-\bar{n} \sin ^{2}\left(\theta^{\prime} / 2\right)}
$$

The graphical plot of this function is very similar to Fig. 1, even for low photon numbers.

For the degree of polarization we get

$$
\mathcal{D}=\frac{3-6 \bar{n}^{2}+8 \bar{n}^{3}+2 \bar{n}^{4}-3 e^{-2 \bar{n}}-6 \bar{n} e^{-2 \bar{n}}}{16 \bar{n}^{2}},
$$

that for large photon numbers $\bar{n} \gg 1$ scales as $\mathcal{D} \simeq \bar{n}^{2} / 8$, which is the same scaling of the number states $|0,0, N\rangle$ in Eq. (5.7).

\section{CONCLUSIONS}

We have presented a natural and consistent approach to the polarization of quantum three-dimensional fields. Due to the noncommuting character of quantum polarization variables, the polarization state must be described always by a probability distribution. We have obtained such a distribution as a marginal of the quadrature $\mathcal{Q}$ function for a three-mode field retaining just the polarization variables. We have found that the states providing the correspondence between quantum states and polarization distributions are the SU(3) coherent states, that are the minimum uncertainty states for the generalized Stokes operators with fixed total number of photons.

We have introduced a degree of polarization as the distance between the polarization distribution and the uniform distribution associated with fully unpolarized light. The approach introduced here is superior to the standard definition in terms of Stokes parameters since it depends on field correlations of all orders. For example, it correctly assesses the polarization state of partially polarized light fields with vanishing Stokes parameters.

We have studied the relation between the 2D and 3D approaches to quantum polarization by considering field states with a component in the vacuum state. We have applied this formalism to some relevant field states such as SU(3) coherent states, quadrature coherent states, and number states.

\section{ACKNOWLEDGMENTS}

I thank Professor J. J. Gil for valuable comments and suggestions. This work has been supported by Project No. FIS2004-01814 of the Spanish Dirección General de Investigación del Ministerio de Educación y Ciencia.

\section{APPENDIX A: STOKES OPERATORS FOR THREE- DIMENSIONAL FIELDS}

The standard two-mode Stokes parameters can be generalized to a three-mode field in the form [6,8-12]

$$
\left\langle S_{j}\right\rangle=\operatorname{tr}\left(\lambda_{j} \Phi\right)
$$

$j=0,1, \ldots, 8$, where $\Phi$ is the $3 \times 3$ correlation matrix for the electric field $\boldsymbol{E}$

$$
\Phi_{j, k}=\left\langle E_{j} E_{k}^{*}\right\rangle
$$

and $\lambda_{j}$ the nine Gell-Mann matrices [the generators of the $\mathrm{SU}(3)$ group]

$$
\lambda_{0}=\left(\begin{array}{lll}
1 & 0 & 0 \\
0 & 1 & 0 \\
0 & 0 & 1
\end{array}\right)
$$

$$
\lambda_{1}=\left(\begin{array}{lll}
0 & 1 & 0 \\
1 & 0 & 0 \\
0 & 0 & 0
\end{array}\right), \quad \lambda_{2}=\left(\begin{array}{ccc}
0 & -i & 0 \\
i & 0 & 0 \\
0 & 0 & 0
\end{array}\right) \text {, }
$$$$
\lambda_{3}=\left(\begin{array}{ccc}
1 & 0 & 0 \\
0 & -1 & 0 \\
0 & 0 & 0
\end{array}\right), \quad \lambda_{4}=\left(\begin{array}{lll}
0 & 0 & 1 \\
0 & 0 & 0 \\
1 & 0 & 0
\end{array}\right) \text {, }
$$

$$
\begin{gathered}
\lambda_{5}=\left(\begin{array}{ccc}
0 & 0 & -i \\
0 & 0 & 0 \\
i & 0 & 0
\end{array}\right), \quad \lambda_{6}=\left(\begin{array}{ccc}
0 & 0 & 0 \\
0 & 0 & 1 \\
0 & 1 & 0
\end{array}\right), \\
\lambda_{7}=\left(\begin{array}{ccc}
0 & 0 & 0 \\
0 & 0 & -i \\
0 & i & 0
\end{array}\right), \quad \lambda_{8}=\frac{1}{\sqrt{3}}\left(\begin{array}{ccc}
1 & 0 & 0 \\
0 & 1 & 0 \\
0 & 0 & -2
\end{array}\right),
\end{gathered}
$$

that satisfy the trace-orthogonality relations

$$
\operatorname{tr}\left(\lambda_{j} \lambda_{k}\right)=2 \delta_{j, k}+\delta_{j, 0} \delta_{k, 0} .
$$

In the quantum domain, the Stokes parameters are the mean values of the Stokes operators [13]

$$
S_{j}=\boldsymbol{a}^{\dagger} \lambda_{j} \boldsymbol{a}
$$

where $\boldsymbol{a}, \boldsymbol{a}^{\dagger}$ are three-dimensional column and row vectors made of the complex amplitude operators and their Hermitian conjugates, respectively.

The generalized Stokes parameters allow us to introduce a degree of polarization for three-dimensional fields. Two alternatives are analyzed in Ref. [5] showing that the most appropriate in the quantum domain is the generalization to three dimensions of the two-dimensional proposal in Ref. [28],

$$
P=\frac{\sqrt{3}}{2} \frac{\sqrt{\sum_{j=1}^{8}\left\langle S_{j}\right\rangle^{2}}}{\sqrt{S_{0}\left(S_{0}+3\right)}} \leqslant \frac{1}{\sqrt{1+\frac{3}{\left\langle S_{0}\right\rangle}}} .
$$

In Ref. [5] it is shown that the maximum degree of polarization $P$ occurs provided that $\Delta S_{0}=0$ and

$$
a_{1}|\psi\rangle \propto a_{2}|\psi\rangle \propto a_{3}|\psi\rangle .
$$

This is satisfied only by the $\mathrm{SU}(3)$ coherent states (B1). 


\section{APPENDIX B: SU(3) COHERENT STATES}

The $\mathrm{SU}(3)$ coherent state $|N, \Gamma\rangle$ can be defined by the action of a $\mathrm{SU}(3)$ transformation on the number state $|0,0, N\rangle[17]$

$$
|N, \Gamma\rangle=e^{i a^{\dagger} R a}|0,0, N\rangle,
$$

where $R$ is a Hermitian $3 \times 3$ matrix, $\Gamma$ represents the parameters required to suitably specify $R$, and $|0,0, N\rangle$ is the product of the vacuum state in modes $a_{1}, a_{2}$ and the number state $N$ in mode $a_{3}$. The above transformation acting on $|0,0, N\rangle$ can be expressed also as a product of two consecutive $\mathrm{SU}(2)$ transformations,

$$
|N, \Gamma\rangle=e^{\theta\left(e^{-i \phi} a_{2}^{\dagger} a_{1}-e^{i \phi} a_{1}^{\dagger} a_{2}\right) / 2} e^{\theta^{\prime}\left(e^{-i \phi^{\prime}} a_{3}^{\dagger} a_{2}-e^{i \phi^{\prime}} a_{2}^{\dagger} a_{3}\right) / 2}|0,0, N\rangle .
$$

This produces the following transformation of the complex amplitude operators:

$$
e^{-i \boldsymbol{a}^{\dagger} R \boldsymbol{a}} \boldsymbol{a} e^{i \boldsymbol{a}^{\dagger} R \boldsymbol{a}}=\mathcal{U} \boldsymbol{a}
$$

where $\mathcal{U}$ is the unitary matrix [19],

$$
\mathcal{U}=\left(\begin{array}{ccc}
c & -s e^{i \phi} & 0 \\
s e^{-i \phi} & c & 0 \\
0 & 0 & 1
\end{array}\right)\left(\begin{array}{ccc}
1 & 0 & 0 \\
0 & c^{\prime} & -s^{\prime} e^{i \phi^{\prime}} \\
0 & s^{\prime} e^{-i \phi^{\prime}} & c^{\prime}
\end{array}\right),
$$

with $c=\cos (\theta / 2), s=\sin (\theta / 2)$, and similarly for the primed variables.

These states are eigenstates of the total number operator $S_{0}=a_{1}^{\dagger} a_{1}+a_{2}^{\dagger} a_{2}+a_{3}^{\dagger} a_{3}$,

$$
S_{0}|N, \Gamma\rangle=N|N, \Gamma\rangle .
$$

Furthermore, it holds that

$$
a_{1}|N, \Gamma\rangle \propto a_{2}|N, \Gamma\rangle \propto a_{3}|N, \Gamma\rangle,
$$

so that the $\mathrm{SU}(3)$ coherent states have maximum degree of polarization $P$ in Eq. (A6) and minimum polarization fluctuations $\delta S$ provided that they are measured as $[5,30]$

$$
\delta S=\sqrt{\sum_{j=1}^{8}\left(\Delta S_{j}\right)^{2}} .
$$

Vice versa, all the states satisfying Eq. (B6) within each subspace of fixed total photon number $S_{0}$ are $\mathrm{SU}(3)$ coherent states.

From Eqs. (B1), (B3), and (B4) the Stokes parameters for $|N, \Gamma\rangle$ are

$$
\left\langle N, \Gamma\left|S_{k}\right| N, \Gamma\right\rangle=N\left(\mathcal{U}^{\dagger} \lambda_{k} \mathcal{U}\right)_{3,3}=N \mu_{k},
$$

for $k=0,1, \ldots, 8$, where the functions $\mu_{k}$ are

$$
\begin{gathered}
\mu_{0}=1, \\
\mu_{1}=2 \sin ^{2} \frac{\theta^{\prime}}{2} \sin \frac{\theta}{2} \cos \frac{\theta}{2} \cos \phi, \\
\mu_{2}=2 \sin ^{2} \frac{\theta^{\prime}}{2} \sin \frac{\theta}{2} \cos \frac{\theta}{2} \sin \phi,
\end{gathered}
$$

$$
\begin{gathered}
\mu_{3}=\sin ^{2} \frac{\theta^{\prime}}{2}\left(\sin ^{2} \frac{\theta}{2}-\cos ^{2} \frac{\theta}{2}\right) \\
\mu_{4}=2 \cos \frac{\theta^{\prime}}{2} \sin \frac{\theta^{\prime}}{2} \sin \frac{\theta}{2} \cos \left(\phi+\phi^{\prime}\right), \\
\mu_{5}=2 \cos \frac{\theta^{\prime}}{2} \sin \frac{\theta^{\prime}}{2} \sin \frac{\theta}{2} \sin \left(\phi+\phi^{\prime}\right), \\
\mu_{6}=2 \cos \frac{\theta^{\prime}}{2} \sin \frac{\theta^{\prime}}{2} \cos \frac{\theta}{2} \cos \phi^{\prime}, \\
\mu_{7}=2 \cos \frac{\theta^{\prime}}{2} \sin \frac{\theta^{\prime}}{2} \cos \frac{\theta}{2} \sin \phi^{\prime}, \\
\mu_{8}=\frac{1}{\sqrt{3}}\left(\sin ^{2} \frac{\theta^{\prime}}{2}-2 \cos 2 \frac{\theta^{\prime}}{2}\right)
\end{gathered}
$$

These functions satisfy an orthogonality relation similar to Eq. (A4),

$$
\int d \Gamma \mu_{j}(\Gamma) \mu_{k}(\Gamma)=\frac{(4 \pi)^{2}}{6}\left(\delta_{j, k}+5 \delta_{j, 0} \delta_{k, 0}\right) .
$$

We note that there is an interesting relationship between quadrature coherent states, $\mathrm{SU}(3)$ coherent states, SU(2) coherent states, and number states, which is displayed in Eqs. (2.7)-(2.9). The three-mode quadrature coherent state $|\boldsymbol{\alpha}\rangle$ is formally identical to a one-mode coherent state, but replacing the number states $|N\rangle$ by the SU(3) coherent states $|N, \Gamma\rangle$ (that are eigenstates of the total number operator $a_{1}^{\dagger} a_{1}$ $+a_{2}^{\dagger} a_{2}+a_{3}^{\dagger} a_{3}$ with eigenvalue $\left.N\right)$. In turn, $|N, \Gamma\rangle$ has the same structure of a two-mode $\mathrm{SU}(2)$ coherent state but replacing the number states $|n\rangle$ in one of the modes by the two-mode $\mathrm{SU}(2)$ coherent states $|n, \Omega\rangle_{1,2}$ (that are eigenstates of the number of photons $a_{1}^{\dagger} a_{1}+a_{2}^{\dagger} a_{2}$ with eigenvalue $n$ ), as shown in Ref. [17]. It is clear that the role played by each one of the modes in this chain of coherent states can be interchanged. Some other examples of this grouping of coherent states can be found in Ref. [29].

There is another expression for the states $|N, \Gamma\rangle$,

$$
\begin{aligned}
|N, \Gamma\rangle= & \frac{\sqrt{N !}}{\left(1+|\xi|^{2}+\left|\xi^{\prime}\right|^{2}\right)^{N / 2}} \sum_{n+n^{\prime} \leqslant N} \frac{\xi^{n^{\prime}} \xi^{n}}{\sqrt{n^{\prime} ! n !\left(N-n-n^{\prime}\right) !}} \\
& \times\left|n, N-n^{\prime}-n, n^{\prime}\right\rangle,
\end{aligned}
$$

where

$$
\xi=\tan \frac{\theta}{2} e^{-i \phi}, \quad \xi^{\prime}=\sqrt{1+|\xi|^{2}} \cot \frac{\theta^{\prime}}{2} e^{i \phi^{\prime}}
$$

In terms of these variables the volume element becomes

$$
d \Gamma=\frac{32}{\left(1+|\xi|^{2}+\left|\xi^{\prime}\right|^{2}\right)^{3}} d^{2} \xi d^{2} \xi^{\prime},
$$

where $d^{2} \xi=d \xi_{r} d \xi_{i}$ being $\xi_{r}, \xi_{i}$, the real and imaginary parts of $\xi$, respectively. This expression can be checked by impos- 
ing the fulfillment of the resolution of the identity in Eq. (2.12).

Furthermore, with this expression the scalar products between $\mathrm{SU}(3)$ coherent states admit a very simple form,

$$
\left\langle N, \Gamma_{1} \mid N, \Gamma_{2}\right\rangle=\left(\frac{1+\xi_{1}^{*} \xi_{2}+\xi_{1}^{\prime *} \xi_{2}^{\prime}}{\sqrt{\left(1+\left|\xi_{1}\right|^{2}+\left|\xi_{1}^{\prime}\right|^{2}\right)\left(1+\left|\xi_{2}\right|^{2}+\left|\xi_{2}^{\prime}\right|^{2}\right)}}\right)^{N}
$$

[1] The Physics of Quantum Information, edited by D. Bouwmeester, A. Ekert, and A. Zeilinger (Springer, Berlin, 2000); H. Paul, Introduction to Quantum Optics (Cambridge University Press, Cambridge, England, 2004).

[2] A. Luis and L. L. Sánchez-Soto, in Progress in Optics, edited by E. Wolf (Elsevier, Amsterdam, 2000), Vol. 41, p. 421.

[3] R. Simon, E. C. G. Sudarshan, and N. Mukunda, Appl. Opt. 26, 1589 (1987).

[4] J. Pollet, O. Méplan, and C. Gignoux, J. Phys. A 28, 7287 (1995).

[5] A. Luis, Phys. Rev. A 71, 023810 (2005).

[6] J. J. Gil, J. M. Correas, P. A. Melero, and C. Ferreira, Proceedings of the 8th Conference Zaragoza-Pau of Applied Mathematics and Statistics, Jaca, 2003; J. J. Gil, J. M. Correas, C. Ferreira, I. San José, P. A. Melero, and J. Delso, Proceedings of the 7 Reunión Nacional de Óptica, Santander, 2003.

[7] J. C. Samson and J. V. Olson, SIAM J. Appl. Math. 40, 137 (1981).

[8] Ch. Brosseau, Fundamentals of Polarized Light: A Statistical Optics Approach (Wiley, New York, 1998).

[9] T. Carozzi, R. Karlsson, and J. Bergman, Phys. Rev. E 61, 2024 (2000).

[10] T. Setälä, A. Shevchenko, M. Kaivola, and A. T. Friberg, Phys. Rev. E 66, 016615 (2002).

[11] M. R. Dennis, J. Opt. A, Pure Appl. Opt. 6, S26 (2004).

[12] S. G. Schirmer, T. Zhang, and J. V. Leavy, J. Phys. A 37, 1389 (2004).

[13] R. D. Mota, M. A Xicoténcatl, and V. D. Granados, J. Phys. A 37, 2835 (2004).

[14] A. Luis, Phys. Rev. A 66, 013806 (2002); Opt. Commun. 216, 165 (2003); Phys. Rev. A 69, 023803 (2004).

[15] M. Kitagawa and M. Ueda, Phys. Rev. A 47, 5138 (1993); D. J. Wineland, J. J. Bollinger, W. M. Itano, and D. J. Heinzen, ibid. 50, 67 (1994).

[16] K. E. Cahill and R. J. Glauber, Phys. Rev. 177, 1857 (1969); 177, 1882 (1969); M. Hillery, R. F. O’Connell, M. O. Scully, and E. P. Wigner, Phys. Rep. 106, 121 (1984); B.-G. Englert, J. Phys. A 22, 625 (1989); H.-W. Lee, Phys. Rep. 259, 147 (1995).

[17] K. Nemoto, J. Phys. A 33, 3493 (2000); K. Nemoto and B. C. Sanders, ibid. 34, 2051 (2001).

[18] A. Luis, J. Phys. A 35, 8805 (2002); Phys. Lett. A 314, 197 (2003).

[19] P. H. Moravek and D. W. Joseph, J. Math. Phys. 4, 1363 (1963).

[20] A. K. Ekert and P. L. Knight, Phys. Rev. A 43, 3934 (1991).

[21] U. Leonhardt and H. Paul, Prog. Quantum Electron. 19, 89 (1995); U. Leonhardt, Measuring the Quantum State of Light (Cambridge University Press, Cambridge, England, 1997); D.-G. Welsch, W. Vogel, and T. Opatrný, in Progress in Optics, edited by E. Wolf (Elsevier Science, Amsterdam, 1999), Vol.
39; N. G. Walker and J. E. Carroll, Electron. Lett. 20, 981 (1984); N. G. Walker and J. E. Carroll Opt. Quantum Electron. 18, 355 (1986); K. Vogel and H. Risken, Phys. Rev. A 40, 2847 (1989); D. T. Smithey, M. Beck, M. G. Raymer, and A. Faridani, Phys. Rev. Lett. 70, 1244 (1993); J. R. Torgerson and L. Mandel, ibid. 76, 3939 (1996); S. Schiller, G. Breitenbach, S. F. Pereira, T. Müller, and J. Mlynek, ibid. 77, 2933 (1996); G. Breitenbach, S. Schiller, and J. Mlynek, Nature (London) 387, 471 (1997); M. Beck, C. Dorrer, and I. A. Walmsley, Phys. Rev. Lett. 87, 253601 (2001); A. I. Lvovsky, H. Hansen, T. Aichele, O. Benson, J. Mlynek, and S. Schiller ibid. 87, 050402 (2001); P. Bertet, A. Auffeves, P. Maioli, S. Osnaghi, T. Meunier, M. Brune, J. M. Raimond, and S. Haroche, ibid. 89, 200402 (2002); A. I. Lvovsky and S. A. Babichev, Phys. Rev. A 66, 011801(R) (2002).

[22] F. T. Arecchi, E. Courtens, R. Gilmore, and H. Thomas, Phys. Rev. A 6, 2211 (1972).

[23] G. S. Agarwal, Phys. Rev. A 57, 671 (1998); J. P. Amiet and S. Weigert, J. Opt. B: Quantum Semiclassical Opt. 2, 118 (2000); S. Weigert, ibid. 6, 489 (2004).

[24] W. Band and J. L. Park, Found. Phys. 1, 133 (1970); J. L. Park and W. Band, ibid. 1, 211 (1971); W. Band and J. L. Park, ibid. 1, 339 (1970); R. G. Newton and B.-L. Young, Ann. Phys. (N.Y.) 49, 393 (1968); A. B. Klimov, O. V. Man'ko, V. I. Man'ko, Y. F. Smirnov, and V. N. Tolstoy, J. Phys. A 35, 6101 (2002); H. F. Hofmann and S. Takeuchi, Phys. Rev. A 69, 042108 (2004).

[25] The distribution for unpolarized light must be invariant under any linear, energy-conserving transformation of the complex amplitudes. This implies that the quadrature $\mathcal{Q}$ function must depend only on $\left|\alpha_{1}\right|^{2}+\left|\alpha_{2}\right|^{2}+\left|\alpha_{3}\right|^{2}$. This is the 3D analog of the 2D problem studied by G. S. Agarwal, Lett. Nuovo Cimento Soc. Ital. Fis. 1, 53 (1971).

[26] E. J. Heller, Phys. Rev. A 35, 1360 (1987); H. Maassen and J. B. M. Uffink, Phys. Rev. Lett. 60, 1103 (1988); I. BialynickiBirula, M. Freyberger, and W. Schelich, Phys. Scr. T48, 113 (1993); A. Lukš and V. Peřinová, Quantum Opt. 6, 125 (1994); B. Mirbach and H. J. Korsch, Ann. Phys. (N.Y.) 265, 80 (1998); A. Anderson and J. J. Halliwell, Phys. Rev. D 48, 2753 (1993); A. Sugita and H. Aiba, nlin.CD/0106012; Č. Brukner and A. Zeilinger, Phys. Rev. Lett. 83, 3354 (1999); M. J. W. Hall, Phys. Rev. A 59, 2602 (1999).

[27] S. Gnutzmann and K. Życzkowski, J. Phys. A 34, 10123 (2001).

[28] A. P. Alodjants and S. M. Arakelian, J. Mod. Opt. 46, 475 (1999).

[29] P. W. Atkins and J. C. Dobson, Proc. R. Soc. London, Ser. A 321, 321 (1971); A. Luis and J. Peřina, Phys. Rev. A 53, 1886 (1996).

[30] R. Delbourgo, J. Phys. A 10, 1837 (1977). 